Review

\title{
Agency, gait and self-consciousness
}

\author{
O.A. Kannape a, O. Blanke a,b,* \\ a Laboratory of Cognitive Neuroscience, Brain Mind Institute, School of Life Sciences, École Polytechnique Fédérale de Lausanne (EPFL), Station 19, 1015 Lausanne, Switzerland \\ b Department of Neurology, University Hospital, 1211 Geneva 14, Switzerland
}

\section{A R T I C L E I N F O}

\section{Article history:}

Received 6 September 2011

Received in revised form 3 December 2011

Accepted 20 December 2011

Available online 4 January 2012

\section{Keywords:}

Agency

Gait

Sensorimotor control

Self-consciousness

Motor awareness

Locomotion

\begin{abstract}
A B S T R A C T
Agency is an important aspect of bodily self-consciousness, allowing us to separate own movements from those induced by the environment and to distinguish own movements from those of other agents. Unsurprisingly, theoretical frameworks for agency such as central monitoring are closely tied to computational models of sensorimotor control. Until recently agency research has largely focussed on goal-directed movements of the upper limbs. In particular, the influence of performance-related sensory cues and the relevance of prediction signals for agency judgements have been studied through a variety of spatio-temporal mismatches between movement and the sensory consequences of movement. However, agents often perform a different type of movement; highly automated movements that involve the entire body such as walking, cycling, and swimming with potentially different agency mechanisms.

Here, we review recent work about agency for full-body movements such as gait, highlighting the effects of performance-related visual and auditory cues on gait agency. Gait movements differ from upper limb actions. Gait is cyclic, more rarely immediately goal-directed, and is generally considered one of the most automatic and unconscious actions. We discuss such movement differences with respect to the functional mechanisms of full-body agency and body-part agency by linking these gait agency paradigms to computational models of motor control. This is followed by a selective review of gait control, locomotion, and models of motor control relying on prediction signals and underlining their relevance for full-body agency.
\end{abstract}

(c) 2012 Elsevier B.V. All rights reserved.

\section{Agency}

The sense of agency describes the feeling of being in control and of being the author of one's movements. In general, one experiences agency, if the outcome of an action corresponds to the outcome one intended. This simple concept closely resembles models of sensorimotor control, which states that in order to successfully control one's movements one has to be able to predict the immediate consequences of one's movements and confirm these predictions against the actual sensory feedback received from the environment. Importantly, these predictions not only allow humans to maintain and correct on-going movements but also to effectively separate their own actions from the environment (Jeannerod, 2003) and from actions of other agents (Daprati et al., 1997; Jeannerod, 2004). One approach to understanding the mechanisms underlying agency, the central monitoring framework (Frith et al., 2000b), is hence based on the internal model for sensorimotor control proposed by Wolpert et al. (Miall and Wolpert, 1996; Wolpert et al., 1995).

The original model, shown in Fig. $1 \mathrm{~A}$, draws on an engineering concept called observer framework and extended earlier thinking

\footnotetext{
* Corresponding author at: Laboratory of Cognitive Neuroscience, Brain-Mind Institute, Station 19, Ecole Polytechnique Fédérale de Lausanne (EPFL), 1015 Lausanne, Switzerland. Tel.: +4121 6939621; fax: +41216939625.

E-mail address: olaf.blanke@epfl.ch (O. Blanke).
}

on oculomotor control and concepts of "corollary discharge" and "efference copies" (Helmholtz, 1866; Sperry, 1950; von Holst and Mittelstaedt, 1950). The observer framework illustrates how i) predictive processing using forward models and ii) actual reafferent feedback are combined to efficiently control and improve sensorimotor control without the need for additional conscious monitoring. The model is generally accepted for sensorimotor control, motor estimation, prediction, and motor learning (Wolpert and Ghahramani, 2000).

How is predictive processing addressed in research paradigms investigating the sense of agency? Following early work by Nielsen (1963, 1978), conscious action monitoring has recently been the topic of intensive research (Fourneret and Jeannerod, 1998; Franck et al., 2001; Knoblich et al., 2004; van den Bos and Jeannerod, 2002). In these studies participants' awareness of their movements was measured in response to sensorimotor mismatches between visual, proprioceptive, and motor signals. The experiments introduced a conflict between the predicted sensory and motor states and the actual state, which relied on deviated feedback. Among others, these studies involved drawing a sagittal line with a stylus (Fourneret and Jeannerod, 1998), retracing a circle (Knoblich and Kircher, 2004), or making pointing movements using a joystick (Franck et al., 2001). Participants were asked to repeatedly perform such goal-directed movements during which their actual hand was hidden from view and replaced by a virtual hand (or equivalent) that could be deviated 
A

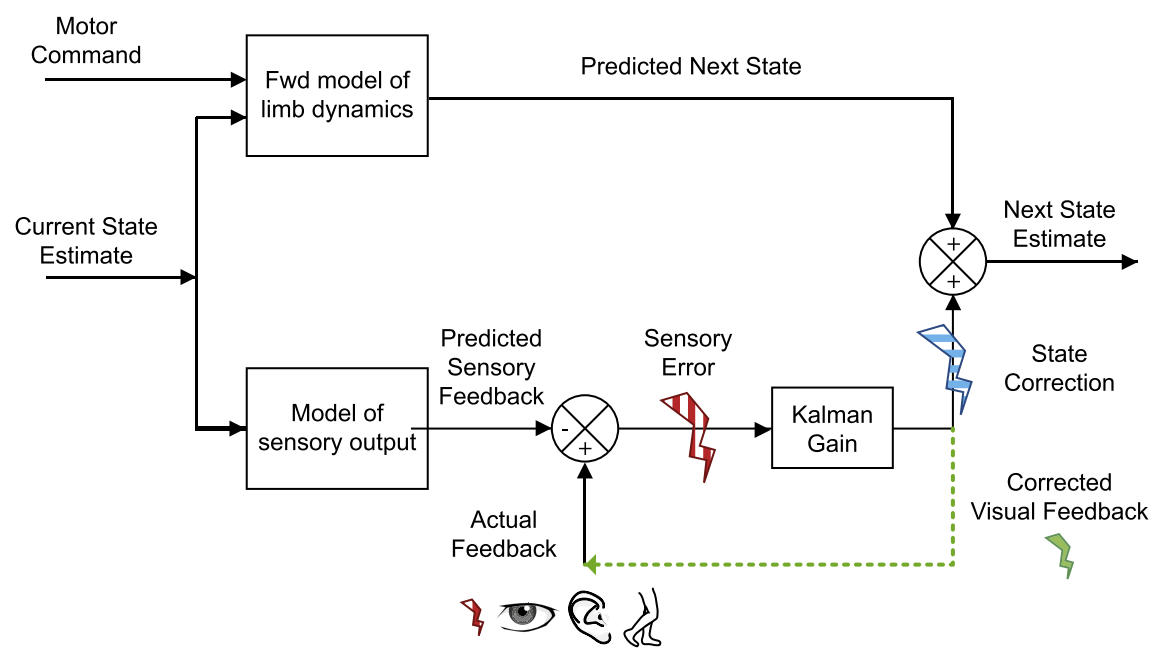

B

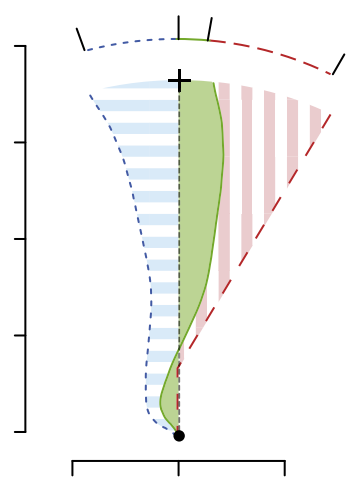

$\square$ experimental deviation
$\square$ motor correction
$\square$ visual feedback

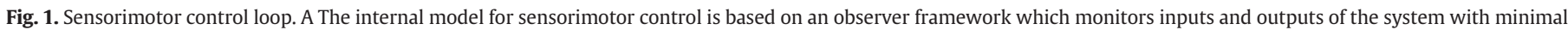

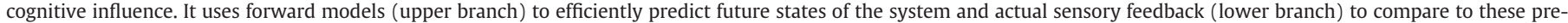

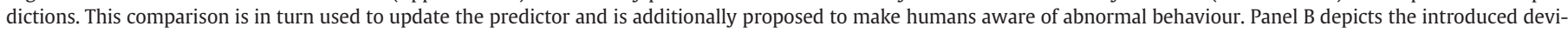

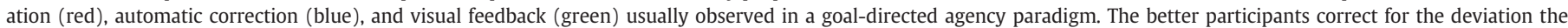

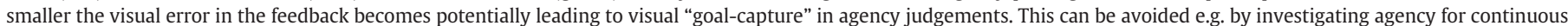
movements such as locomotion.

in space or delayed in time. At the end of each trial participants judged whether the movement they had seen corresponded to the movement they had just performed. Psychometric thresholds were subsequently extracted from these responses in order to determine the spatio-temporal limits of agency.

Apart from measuring participants' sense of agency for their movements, these studies were important as they illustrated that participants automatically compensated for a displayed spatial deviation in order to align their hand trajectories with the visual target on the computer screen. The participants were often unaware of their online corrections and judged many of these actions as nondeviated. As we will discuss throughout this review it is important to closely inspect both motor awareness and the accompanying motor corrections and their influence on motor awareness as such motor corrections change the initially presented spatio-temporal deviation amplitudes. Unlike that of agency there is no standard measure for motor corrections, which in addition depends on the selected agency paradigm. Motor corrections have thus been variably based on the squared RMS (root-mean-squared) deviation from an ideal trajectory (Fourneret et al., 2001), mapping of polar coordinates during circle drawing (Knoblich and Kircher, 2004) or the amount of spatial deviation compensated at the end of each trial (Kannape et al., 2010). Detailed motion analysis in combination with agency judgements will be crucial as it allows comparing the actual state of the participant's motor system to the deviated feedback he receives. Motor corrections reflect how the current estimate and the predictions of future motor states are affected by the feedback and whether such motor corrections precede motor awareness (agency). Analysing motor performance across several experimental conditions is further necessary in order to relate it to the prevalent theoretical frameworks for agency (Blakemore et al., 1998a; Frith, 2005) and the aforementioned computational models of sensorimotor control (Todorov and Jordan, 2002; Wolpert et al., 1995).

Research on the sense of agency illustrated that participants selfattributed their own movements (i.e. experienced a sense of agency for their own movements) for feedback that was deviated in space by up to $15^{\circ}$ even though they unconsciously changed their movement trajectories. Analogously, Franck et al. (2001) investigated the influence of temporal cues on agency judgements. The authors introduced different temporal delays between the visual position of the participant's hand (as seen on a computer screen) and the participant's actual hand while carrying out a pointing task. As observed for spatial deviations, increasing delay durations between visual and sensorimotor cues were found to decrease agency judgements (see also Shimada et al., 2010) with subjects self-attributing delayed movements below delays of $150 \mathrm{~ms}$. These studies extended earlier work by Castiello et al. (1991) showing that the generation of a goal-directed action and the building of a conscious experience thereof are closely related, but distinct, processes.

Agency abnormalities have been observed in patients suffering from schizophrenia, in whom abnormal action attribution (agency) may include a variety of passivity symptoms, in particular delusions of control (Schneider, 1955), in which such patients may attribute agency for own actions to an external agent (or vice versa, as reviewed in Fourneret et al., 2001; Frith et al., 2000a; Jeannerod, 2009). As sensorimotor control, perception, and action execution are generally intact in these patients, it has been proposed that agency abnormalities in schizophrenia arise due to action attribution deficits (Jeannerod, 2009) resulting from cortical deficits in predictive signal integration. Such deficits have also been proposed to be of relevance for neurologically-induced abnormalities in action-awareness and sensorimotor control such as the so-called "alien hand syndrome", during which movements of a body part, mostly an upper limb, are perceived as generated by another or alien person (Feinberg et al., 1992).

These clinical examples illustrate the importance of predictive processing for the sense of agency and for maintaining a coherent representation of the self as a causal agent. Similarly, previous investigations of agency were important for the study of bodily selfconsciousness, but were focussed on agency for actions of fingers (Knoblich and Repp, 2009; Repp, 2005, 2006; Repp and Knoblich, 2007), hands (Daprati et al., 1997; Daprati and Sirigu, 2002; Salomon et al., 2011; van den Bos and Jeannerod, 2002), or arms (Fourneret and Jeannerod, 1998; Franck et al., 2001; Knoblich and Kircher, 2004; Nielsen, 1963; Synofzik et al., 2006). As the participants' body position was kept constant (except for actions of finger, hand, or arm, as indicated in Fig. 2A), these studies did not investigate agency for more global aspects of the bodily self that have been studied with respect to the related concept of full-body ownership (self-identification, self-location, and the first-person perspective; Blanke and Metzinger, 2009; Ehrsson, 2007; Lenggenhager et al., 
A

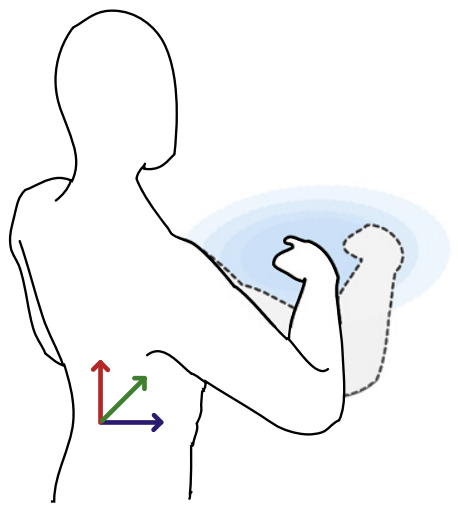

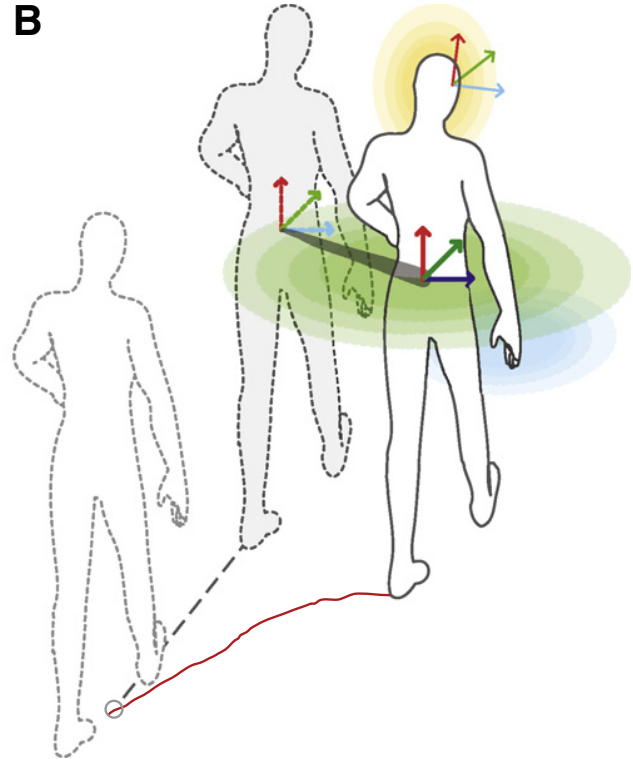

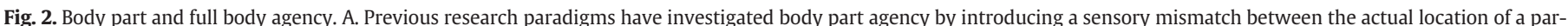

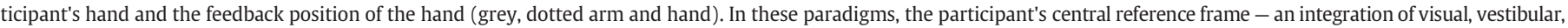

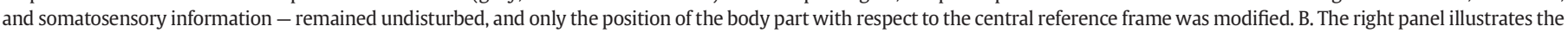

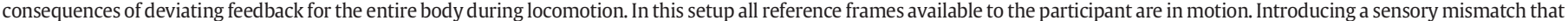

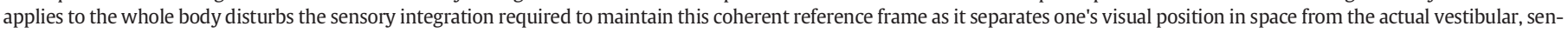
sorimotor position, in effect separating or blurring one's self-localisation in space.

2007). Full-body ownership and full-body agency are associated with the position and movement of the entire body of the participant as is the case during locomotion. In addition to differences in the involved sensorimotor systems between locomotion and full-body agency (lower limbs; bilateral movements) versus body-part movements and agency (upper limbs; unilateral movements), locomotion also gives rise to vestibular sensations and changes in the perception of the surrounding extrapersonal space, Fig. 2B, differing from changes during arm movements in stable or sitting actors, Fig. 2A. Locomotion further differs from upper limb actions in physiological ways: Gait is cyclic, more rarely immediately goal-directed, and is generally considered a highly automatic and unconscious action with important control centres in spinal cord and brainstem (Armstrong, 1988; Grillner and Wallen, 1985). Based on these differences the sense of agency could be expected to differ between upper-limb and full-body movements. Investigating locomotion is therefore not only relevant for the study of bodily self-consciousness but also for understanding agency processing for a new set of continuous movements involving the entire body. We here discuss two novel gait agency paradigms in detail. In the subsequent chapters we relate these to the internal model of sensorimotor control and the role of predictive processing.

\subsection{Spatial, visuo-motor conflicts and full-body agency}

The first experiment to measure gait awareness, and therefore fullbody agency (Kannape et al., 2010) was based on an original paradigm by Nielsen (1978) and extended a more recent experiment by Fourneret and Jeannerod (1998) (see Fig. 3A). Kannape et al. (2010) provided visual feedback by tracking the participants' movements and playing them back in real-time with the use of full-body motion capture and virtual reality technology. Participants were asked to walk their virtual body (shown on a large projection screen in front of them, Fig. 3A) into a virtual target cylinder in a straight line. During some of these trials a visual sensorimotor conflict was introduced by deviating the walking trajectory of the virtual body to either the left or the right by $5^{\circ}, 10^{\circ}, 15^{\circ}$, or $30^{\circ}$. In order to reach the targets, participants had to compensate for the introduced deviation by correcting their walking trajectory in the opposite direction (coloured walking trajectories, Fig. 3B).

This setup allowed quantifying the precision with which the participants control and monitor the displacements of their entire body in extrapersonal space. The findings show that participants track the position and locomotion of their body with a low accuracy within a peripersonal space of $10-15^{\circ}$. As illustrated in Fig. 3C, deviations of $5^{\circ}, 10^{\circ}$, and $15^{\circ}$ lead to many erroneous self-attributions (in $29.2-82.9 \%$ of all trials) although participants correctly changed their gait in order to reach the virtual target positions. This incorrect gait agency during goal-directed locomotion and navigation suggests that the conscious monitoring of an agent's moving body is not reliable and that the generation of full-body locomotion and the building of a conscious experience of it are distinct brain processes. The value of $10-15^{\circ}$ above which angular biases during cyclic locomotion were more often correctly perceived than not by our participants (no selfattribution) was compatible with earlier work on agency for goaldirected hand and arm movements (thresholds of $6.5^{\circ}-15^{\circ}$; Farrer et al., 2003; Franck et al., 2001; Jeannerod and Pacherie, 2004; Posada et al., 2007; Slachevsky et al., 2001; Synofzik et al., 2006).

Importantly the participants' actual walking trajectories illustrated that they automatically compensated for introduced spatial mismatches, even if they were not aware of such a mismatch, as indicated by the agency judgement (Fig. 3B). In trials with deviations of $15^{\circ}$, corresponding to the agency threshold, participants still automatically corrected their walking trajectories by $7^{\circ}-8^{\circ}$. For the largest deviations of $30^{\circ}$ participants reported a switch in strategy in that they now consciously corrected their walking trajectories in order to complete the task. Overall, participants compensated about $40 \%$ of the introduced mismatch, meaning only $60 \%$ of the original mismatch remained in the visual feedback.

A direct consequence was that the deviation in the visual feedback became harder to detect, the better participants compensated for it 
A

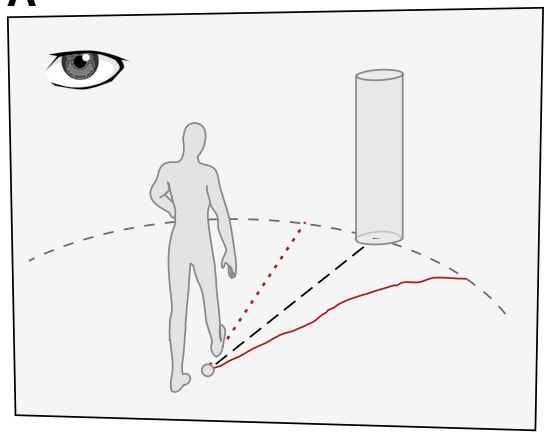

\section{B}

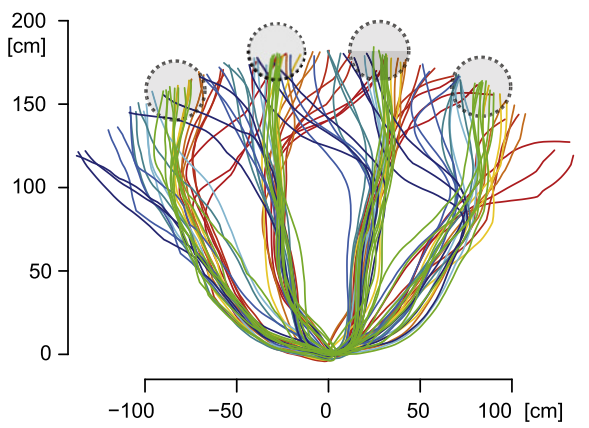

C

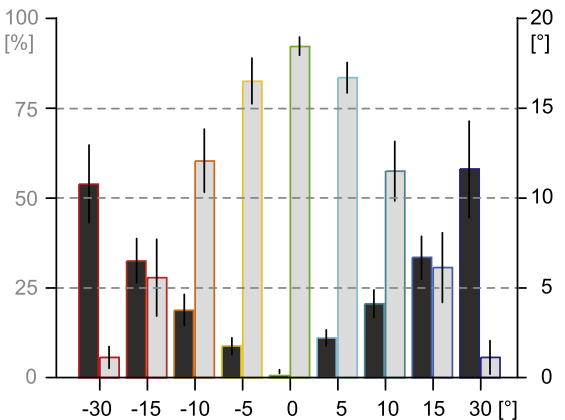

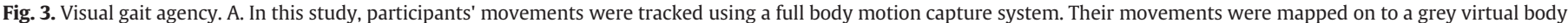

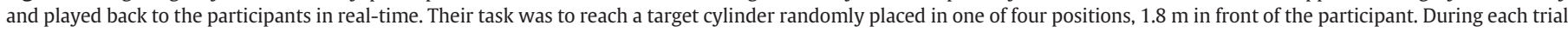

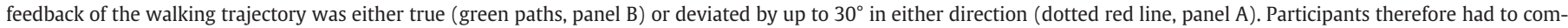

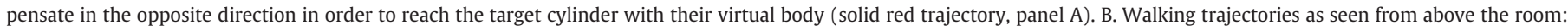

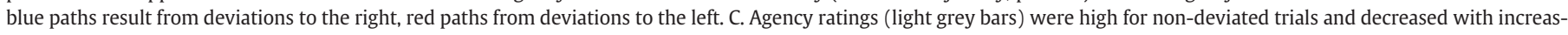

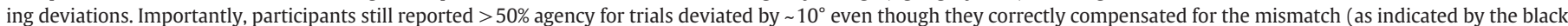
bars).

(cf. Fig. 1B). Kannape et al. (2010) therefore introduced the Motor Awareness Index (MAI) to relate agency judgements to the sensorimotor corrections in one term. The MAI allows comparing agency thresholds across participants (and across experimental conditions) that do not compensate for the same deviations to the same extent. For example, if a participant does not correct his trajectory to compensate for a spatial deviation he would not reach the target, his actual position would not correspond to the predicted position, and it would be easier for that participant to detect the mismatch based on the larger visual error. For someone who entirely compensates the spatial deviation, the visual feedback contains no error and matches the predicted position. Agency judgements are therefore based on different sensorimotor signals in these trials. The MAI addresses the sensitivity of agency to the predicted visual consequences of one's movements with respect to the actual movement. Furthermore, a high MAI indicates that the sensorimotor control of full-body movements and agency for these movements may be distinct processes because participants would even rate movements with a strong sensorimotor conflict (caused by compensating for larger angular deviations) as self-generated. The MAI allows determining how the otherwise inaccessible relationship between the initially presented angular deviation, the resulting motor prediction and the actual motor correction influenced agency.

Despite the different functional consequences of the movement of a person's body part or a person's entire body, these experimental data suggest that humans rely on comparable mechanisms for body-part agency (i.e. arm) and agency for movements involving the entire body such as locomotion. The authors provide additional evidence in support of effector-independent mechanisms for agency in a second study, by combining the gait agency paradigm with two classical effects from the literature that investigates 1 ) the processing of upright bodies (inversion) and 2) of the visuo-spatial perspective (direction). Previous research on the perception of body postures (Reed et al., 2003) and biological motion (Cutting et al., 1988), as well as body-part agency (Knoblich and Kircher, 2004; Knoblich et al., 2004), has observed a significant decrease in the performance of perceptual tasks for inverted stimuli (body postures or biological motion), the so-called inversion effect (1). Similarly, agency judgements and other performances decreased for stimuli rendered from a non-egocentred visuo-spatial perspective (David et al., 2006; Vogeley and Fink, 2003), e.g. a $180^{\circ}$ rotation with respect to an arm movement (van den Bos and Jeannerod, 2002) (2). Comparable mechanisms have also been reported for the related sense of body ownership for the upper extremity (Tsakiris and Haggard, 2005) (perspectival direction effect).
Kannape et al. (2010) found that gait agency (without taking into account on-going gait movements during the task) was not directly influenced by orientation and direction of the visual feedback. Thresholds slightly increased but not at a significant level. However, participants' gait movements changed significantly and were most accurate for familiar, upright feedback, indicating once more that automatic sensorimotor correction was more accurate than the participants' sense of agency. These two findings were reflected in the MAI analysis, which revealed that taken together, the actual angular displacement that resulted from the gait correction in each experimental condition strongly affected gait agency, for inverted virtual bodies leading to higher MAI values. Hence, larger sensorimotor mismatches were necessary in the inverted conditions before participants became aware of them. Taken together, these two studies suggest that the sense of agency for full-body movements resembles that for actions of individual body-parts as it is correspondingly affected by angular deviations and stimulus inversion. Gait agency was not affected by the perspectival direction of the feedback, perhaps due to the ecological familiarity with reflections as in a mirror or shop window. While further research is required, these data indicate that the mechanisms underlying agency are independent of the effector involved, at least for visual, goal-directed actions.

\subsection{Temporal, auditory motor conflicts and full-body agency}

Most previous work on agency has focussed on the investigation of performance-related visual cues (Daprati et al., 1997; Farrer et al., 2003; Fourneret and Jeannerod, 1998; Franck et al., 2001; Knoblich and Kircher, 2004; Salomon et al., 2011; Shimada et al., 2010; Tsakiris and Haggard, 2005; van den Bos and Jeannerod, 2002). Yet, the majority of our actions have consequences that are apparent across multiple sensory modalities including touch and audition. Do mechanisms of agency differ when another sensory cue signals action consequences? Auditory action consequences may differ in their effects from visual action consequences, as the auditory detection of temporal discrepancies may be an especially powerful agency cue due to the excellent timing of auditory perception. Yet, such auditory effects on agency have only rarely been tested (Asai and Tanno, 2008; Knoblich and Repp, 2009; Repp and Knoblich, 2007; Sato, 2008; Sato and Yasuda, 2005). In addition, analogous to the visual paradigms described above, most agency studies with auditory feedback have tested body-part agency.

However, footstep-related auditory signals during locomotion are probably one of the most common performance-related auditory cues (alongside speech or eating) and likely of relevance for bodily self- 
consciousness. Concerning gait, auditory cues have been shown to alter gait (in Parkinson's Disease, Enzensberger and Fischer, 1996; healthy elderly, Roerdink et al., 2011; stroke rehabilitation, Thaut et al., 1997), but their influence on gait agency is not well understood. This was only recently studied by Menzer et al. (2010) who investigated gait agency by introducing temporal conflicts between footsteps and footstep-related auditory cues. In this free-walking paradigm participants wore specially adapted shoes that were equipped with open microphones. Through these shoes participants received auditory feedback of their footsteps at different delays while walking continuously in a $20 \mathrm{~m}$ by $4 \mathrm{~m}$ hallway. Each trial lasted $7 \mathrm{~s}, 11$ steps on average, during which participants received feedback in real-time $(16 \mathrm{~ms})$ or with one of 19 random delays of up to $1800 \mathrm{~ms}$ (see Fig. 4A, cf. Menzer et al., 2010, for full details).

In-line with previous reports on body-part agency, participants gave the strongest self-attributive gait agency judgements (>90\%) in trials with the smallest temporal delays between 16 and $100 \mathrm{~ms}$. Gait agency decreased to 34\% and 28\% for delays between 250 and $450 \mathrm{~ms}$ respectively. Gait-corrected agency judgements, shown in Fig. 4C, illustrate the systematic and predictable relationship between agency judgements and the extent of the auditory delay: gait agency ratings periodically peaked when the duration of the auditory delay matched the duration of the participants' step-cycle. This was the case for delays of a full step-cycle ( 1 on the abscissa) as well as delays of a single step and three steps ( 0.5 and 1.5 on the abscissa). In these latter conditions, simultaneous to taking a left footstep, participants heard the auditory signal associated with a previous right footstep. Participants hence could not distinguish between the sound of their current foot-step and the sound of a previous foot-step, even if this was produced by the contralateral foot.

Participants walked with an average step-cycle of $1.25 \mathrm{~s}$ ( \pm 130 ms SD; range: $1.01-1.79$ s). Surprisingly, their step-cycles varied systematically with the delay period in a sinusoidal pattern (Fig. 4B). On average individual footsteps were separated by about $600 \mathrm{~ms}$, compatible with physiological data in healthy subjects (Blanc et al., 1999; Macellari et al., 1999). Fig. 4B depicts that the step-cycle (walking speed) showed small but systematic variations as a function of the delay conditions. A first maximum occurred for $\sim 400 \mathrm{~ms}$, followed by a minimum for $600 \mathrm{~ms}$, a maximum for $900 \mathrm{~ms}$, another minimum for $1200 \mathrm{~ms}$, and a final maximum for $1500 \mathrm{~ms}$. The delay conditions with smallest step-cycle (or fastest walking speed) overlapped with the delay conditions for which gait agency judgements reached maximal values (compare with Fig. 4C), comparable to those at the minimal delay $(16 \mathrm{~ms})$. These data further exposed automatic changes in gait that depended on sensorimotor conflicts by showing that participants' walking speed depended on the delay period, increasing after each stride and decreasing at each stride, although they were not aware of this and were instructed to walk at a comfortable speed throughout the experiment.

The temporal thresholds reported by the authors for these audiomotor cues were similar for judging delays between visuo-motor cues, as reported for studies on body-part agency. This was found even though the temporal resolution of the auditory system is higher than the temporal resolution of the visual system. The delay values of 100-200 ms are far above the threshold for auditory temporal order judgements of $\sim 20 \mathrm{~ms}$ (Hirsh, 1959; Hirsh and Sherrick, 1961; cited in Knoblich and Repp, 2009). We therefore suggest that this similarity reflects common, modality-independent or supramodal, mechanisms in agency for auditory and visual gait consequences. This is compatible with a comparison of the predicted and actual consequences of actions and gait (Frith et al., 2000b) that has been proposed to be independent of the sensory modality tested. However, given the relative rarity of studies testing the effects of performance-related auditory cues on agency (especially gait agency) and the lack of studies comparing the effects of visual and auditory cues on agency directly in the same subjects, this has to be regarded with caution and may depend on the employed agency manipulation and task and may further differ between explicit and implicit agency judgements (Repp and Knoblich, 2007). Nonetheless the study by Menzer et al. (2010) yielded comparable effects to the visual-motor paradigm mentioned above suggesting that the sense of agency is both supramodal and effector independent processes including cyclic full-body actions such as gait.

\section{Predictive processing in agency}

Since its introduction the observer framework for sensorimotor control has been adapted and extended to the concept of central monitoring in order to account for cognitive disorders, schizophrenic symptoms (Frith et al., 2000a), a range of abnormalities in motor awareness (Blakemore et al., 2002; Frith et al., 2000b) and as a neurocognitive model of agency as a whole. In the following we will discuss the approach of previous agency paradigms with respect to the original internal model for sensorimotor control as proposed by Wolpert et al. (1995) and argue that this suffices to explain most of the observed automatic corrections. However, the novel findings from research on gait agency reviewed here imply that a simple expansion of this model cannot account for an understanding of all aspects of the sense of agency. Continuous full-body movements can be used to challenge the central monitoring framework and to emphasise the pre-reflective aspects of agency, sometimes referred to as a "feeling of agency" (David et al., 2008; Synofzik et al., 2008).
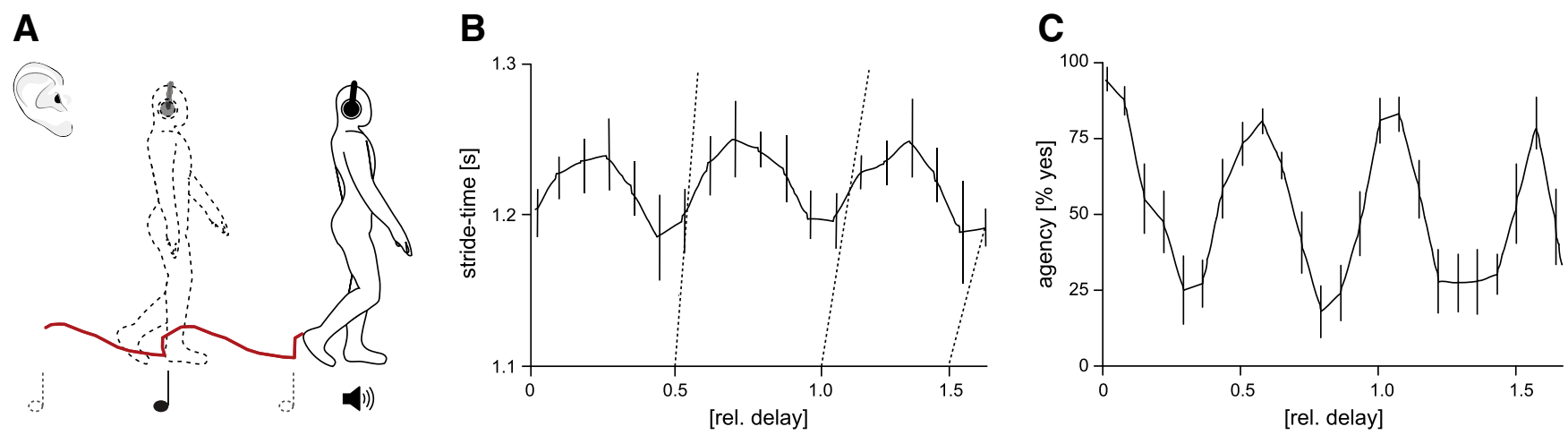

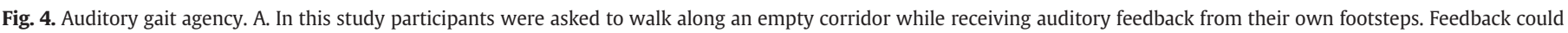

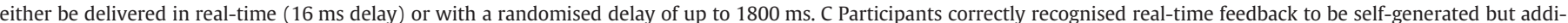

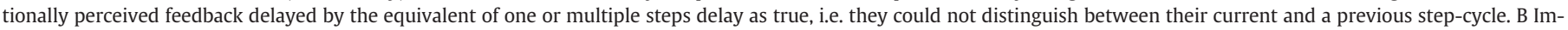

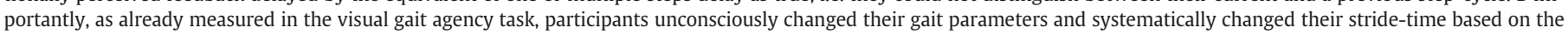
delay of the auditory feedback. 


\subsection{Automatic sensorimotor control}

When we perform an action our movement intention, or immediate goal, is translated into a motor command by an internal inverse model, often referred to as a controller. This controller translates an abstract goal into patterns of joint movements and muscle activations. An efference copy of the motor command is produced and used to predict the sensorimotor consequences of the ensuing movement. This so-called forward model predicts the next state of the system given the current state and the efference copy (upper branch in Fig. 1A). In the forward model, this happens before any sensory feedback is available. One advantage of the forward model is that movements can be planned and adjusted without the need to wait for sensory feedback which can be delayed by up to $250 \mathrm{~ms}$ (Miall et al., 1993), which is one of the reasons why such models have first been proposed for ballistic movements, such as eye movements (Sperry, 1950; von Holst and Mittelstaedt, 1950). Simultaneously, the sensory consequences of the action are predicted using a sensory forward model (lower branch, Fig. 1A) and is for example used to attenuate the sensory consequences following selfinitiated movements. The predicted sensory feedback is compared to the actual, re-afferent sensory feedback. Small mismatches between predicted and actual feedback are subsequently used to update the current state estimate. The crucial comparator mechanism then compares the predicted state of the motor system with its corrected, actual estimate to improve the predictor and correct an on-going movement.

Evidence in favour of the first comparator (comparison between predicted and actual sensory feedback) stems from a study by Blakemore et al., who showed - based on earlier work by Weiskrantz et al. (1971) that humans attenuate the sensory response to self-generated stimulation as illustrated by the fact that one cannot tickle oneself (Blakemore et al., 1998b, 2000). Evidence in favour of the second comparator (comparison between predicted and actual states of the motor system) and the understanding that the internal model of sensorimotor control functions as an optimal state estimator is provided by Wolpert et al. (1995). In this task participants were asked to estimate the position of their hand after performing a reaching task, undisturbed or against imposed external forces and without visual feedback. These data strongly favour an internal model of sensorimotor control that makes trade-offs between an internal forward model and actual sensory feedback. This second comparator has recently received much attention for its proposed role in bodily self-consciousness as it is assumed that the sense of agency crucially depends on its output.

\subsection{Movement correction and agency}

The paradigms introduced by Torsten Nielsen and later by Marc Jeannerod and colleagues were important as they quantified the limits of conscious access humans have to these sensorimotor control mechanisms. Thus, for upper hand movements participants were not aware of deviations of up to $15^{\circ}$ between the position of their actual occluded hand and the position of their seen virtual hand, even though they automatically corrected for these deviations. This correction is compatible with the aforementioned control mechanism, as participants non-consciously adjusted their movement trajectories based on discrepancies between predicted and actual state estimate which was experimentally deviated in space or time. This suggests that the comparator mechanism is accurate enough to correct small deviations automatically but that one does not have conscious access (or insight) into this process. Instead, this mechanism controls for the many smaller perturbations and only creates a conscious break in agency, if sensory mismatches become too large to be considered a false alarm.

Two open questions resulted from this line of research: 1) what are the limits and complexity of motor compensation (state correction) we may perform unconsciously and 2) which error cue is more important to the sense of agency - the experimentally induced error in audio-visual feedback or the state-correction within the sensorimotor control loop. In previous studies these questions were often not addressed either because participants never became fully aware of their corrective motor performance (e.g. Fourneret and Jeannerod, 1998; Nielsen, 1963) or because the movement data was not discussed relative to the agency ratings (or simply unavailable, e.g. Franck et al., 2001; Leube et al., 2003).

Investigating agency using locomotion (gait agency) has provided insights into both of these questions. First and with respect to the complexity of the movements, the studies highlighted in this review illustrate that we are able to automatically adjust our gait, similar to adjustments for movements of the upper limbs although gait agency can differ as seen for a perspectival change in feedback. Apart from the complexity of this movement, human locomotion differs from reaching movements in other ways. Walking towards a target destination $2 \mathrm{~m}$ ahead or along a $20 \mathrm{~m}$ corridor requires a series of coordinated motor-sequences as opposed to the ballistic movements made for example in a pointing task (cf. Bridgemen et al., 1981). This suggests that in order to successfully complete this task, gait corrections are subdivided and coordinated literally step by step.

Next to the complexity of corrective gait movements, these locomotion paradigms also delineate the limits of automatic sensorimotor correction. In order to compensate for the largest delays in the goaldirected walking paradigm (Kannape et al., 2010) for example, participants reported consciously adjusting their walking trajectories similar to the switch in motor-compensation reported in Slachevsky et al. (2001, 2003) for arm-movements . This change in strategy occurred for deviations of about $15^{\circ}$ and motor compensation of about $7^{\circ}-8^{\circ}$. It is therefore possible that this threshold, which marks a maximum "leeway" for automatic sensorimotor control, is used as an indicator that an action is no longer fully controlled by oneself and directly impacts the experience of agency. As will be discussed in the next section, the magnitude of this threshold determines the remaining visual error in the feedback (Fig. 1B) and may point to a potential bias inherent in goal-directed agency paradigms which can be avoided by investigating agency for continuous movements.

The notion that agency judgements may be based rather on the sensorimotor conflict introduced by automatic compensation then the introduced visual/auditory conflict in the feedback is further supported by a series of studies by Knoblich and Kircher (2004) in which participants performed a drawing task during which the relative velocity between their actual movement and its visual consequences was perturbed. In one of the conditions participants were asked to stop drawing as soon as they detected the mismatch. The results confirmed that motor correction always preceded conscious awareness of a mismatch. Varraine et al. (2002) demonstrated partly analogue results for treadmill walking. In this experiment participants unconsciously adjusted their walking characteristics, force or speed, for about $6 \mathrm{~s}$ even though they were explicitly asked to report these changes as soon as they arose (although this delayed awareness may result from a difference in the paradigm as perturbations were only increased gradually and no visual feedback was available to the participants). Data from a deafferented patient further supports that agency judgements may be based on the sensorimotor compensation as opposed to the visual feedback. This patient correctly compensated for visually represented spatial deviations, but - due to lack of reafferent haptic signals - never became aware of the deviations or the own motor correction (Fourneret et al., 2002). Even though the prediction and updating of sensorimotor control were accurate, the actual estimate of the current state was solely based on the visual feedback, which matched the state intended by the patient. Taken together these findings underline the necessity to closely analyse participant movements in agency studies and relate them systematically to their conscious reports of agency. For one, they give insight into the magnitude and complexity of motor corrections that are performed automatically and point to dissociations between agency and motor 
control under normal conditions. For another they illustrate that once motor corrections pass a threshold, such as the $7^{\circ}-8^{\circ}$ in the goaldirected walking paradigm (during trials with a visual deviation close to the threshold, i.e. $15^{\circ}$ ), this dissociation is bridged and we become aware of the sensorimotor corrections.

\subsection{Agency and sensorimotor control for continuous movements}

All of these agency paradigms illustrate that small error signals, environmental noise and even experimentally induced deviations are seamlessly integrated to update and correct one's on-going movements (Faisal et al., 2008). However, it has been suggested that the goal-directedness of these studies presents a cognitive bias, which causes one to remain largely unaware of conflicting sensory feedback about the actual state of one's motor system as long as one's intended goal has been achieved (Blakemore et al., 2002). Unlike movements in previous reaching, grasping, and drawing tasks locomotion is inherently continuous and often not immediately goal-directed. Investigating gait agency can therefore avoid this cognitive influence of task-completion. The foot-step related auditory experiment on gait agency particularly underscores this point. Participants were performing a continuous walking task that was not goal-directed. Nonetheless they integrated the continuous auditory feedback of their foot-steps into the sensorimotor loop as illustrated by the change in walking speed and this change was independent of the participants' agency judgements. According to the comparator model the delayed feedback causes a mismatch between the predicted sound of the footstep and the actual feedback. This sensory error will be used to correct the estimated state of the sensorimotor system, see Fig. 1A (Wolpert et al., 1995). This state correction would propagate that the timing of the current state estimate was inaccurate and result in an adaptation of timing and therefore walking speed. This is supported by the fact that the amount of change in walking speed observed by Menzer et al. (2010) corresponded to the level of mismatch between predicted and actual feedbacks. This hypothesis is further supported by two recent experiments which showed that delayed haptic gait feedback (vibration of the foot soles, Watanabe and Ando, 2010) as well as delayed visual feedback of one's own gait (Kannape and Blanke, 2010) caused participants to systematically adjust their walking speed. Thus, participants integrated the performance-related feedback independent of whether or not participants perceive the same feedback to be self-generated.

An important novelty introduced by these paradigms is that the gait movements could change independently of the agency ratings. In previous goal-directed reaching tasks, agency always closely followed the magnitude of the visual mismatch and the strength of the resulting motor correction. Yet, the data reviewed here show that gait agency and gait movements can change separately as the observed gait changes are to some extent task-irrelevant (Menzer et al., 2010; Kannape and Blanke, 2010). This is also of interest for the central monitoring theorem (Frith et al., 2000b), as in this case, agency judgements can neither rely on the success or failure in reaching a target nor can they be based on the amount of underlying motor correction. Synchronising the gait to the underlying rhythm could be a means to minimising uncertainty about whether or not one is the agent responsible for the received feedback (Wolpert et al., 2011). If gait was temporally synchronous to the feedback, even if left-right reversed, the (temporal) error in the motor-system would be cancelled out. Accordingly, uncertainty about agency would be limited to spatial differences in visual feedback or qualitative differences for auditory feedback.

\subsection{The neuroscience of gait and agency}

Although gait has initially been considered a largely automatic action regulated mainly by subcortical control mechanisms including the brainstem and spinal cord (Armstrong, 1988; Grillner and Wallen, 1985), recent work has highlighted the influence of attention, executive function and other cognitive mechanisms (Hausdorff, 2005; Shaw, 2002; Yogev-Seligmann et al., 2008). Walking is a complex task involving the integration of locomotion, balance, and adaptation in an ever-changing environment (Armstrong, 1988; Blanc et al., 1999; Drew et al., 2004). However, the neuroscience of human upright gait is hampered by two main caveats. Neuroimaging using functional magnetic resonance imaging (fMRI), magnetoencephalography (MEG), or electroencephalography (EEG) is currently not available or severely limited in walking humans (Fukuyama et al., 1997; Miyai et al., 2001) and humans are the only truly upright walking primates (Eccles, 1989). Only recently Independent Component Analysis and template based artefact removal have been applied to EEG recordings to recover and examine electrocortical activity coupled to the human gait-cycle (Gwin et al., 2011). More work along these lines is mandatory. The few studies that have been conducted point to a distributed network for locomotion including spinal cord, brainstem, basal ganglia, cerebellum, motor and posterior parietal cortices (for motor cortex see Armstrong, 1988; Drew et al., 2004; for spinal cord: Grillner and Wallen, 1985; Nutt et al., 1993).

Despite these recent efforts, neuroscience of walking is thus almost entirely based on findings in quadrupeds and behavioural work in patients with gait disorders and is currently absent for gait agency. One relevant group to investigate both with respect to locomotion as well as the sense of agency and voluntary motor control are patients suffering from Parkinson's disease. Gait-impairments such as asymmetry or freezing-of-gait are well documented (Browner and Giladi, 2010; Morris et al., 1994), as is the underlying pathology (Agid and Blin, 1987; Alexander et al., 1986), but beyond the scope of the current review. Although initial work on agencyrelated processing in Parkinsonism has been published by Moore et al. (2010b), further research is required to systematically link such findings to models of sensorimotor control, locomotion, and upperlimb and gait agency. We also mention that upper-limb agency has been addressed in a number of functional brain imaging studies revealing a distributed network of involved brain areas (reviewed by David et al., 2008) that includes supplementary motor area (Farrer and Frith, 2002; Moore et al., 2010a), the cerebellum (Blakemore et al., 2001) but also associative cortices, i.e. dorsolateral prefrontal cortex (Fink et al., 1999), posterior parietal cortex (Farrer et al., 2003; Farrer and Frith, 2002), posterior superior temporal sulcus (Leube et al., 2003) and the insula (Cunnington et al., 2006).

The gait agency paradigms we have reviewed here are attractive for investigating agency for a number of reasons. As mentioned above, locomotion is a complex but highly practised, largely automatic action that involves the entire, spatially situated body. Unsurprisingly, similar to the observer framework for sensorimotor control, locomotion is mostly regulated unconsciously and is rarely immediately goaldirected. Investigating gait agency using locomotion can therefore remove confounds such as reaching a target location as could the study of agency for finger tapping-movements (i.e. Knoblich and Repp, 2009; Repp and Knoblich, 2007). Furthermore, the study of gait agency opens up the possibility of applying paradigms such as dual tasking to investigate bodily self-consciousness and the sense of agency and related functions of spatial navigation and path integration (Glasauer et al., 2009; Loomis et al., 1993; Wolbers and Hegarty, 2010). This is important as humans may perform many perceptual, motor, and cognitive tasks while walking, potentially causing performance in these tasks to decline or change. Additionally these secondary tasks have been shown to alter gait characteristics and even lead to freezing or falls (for comprehensive reviews see Woollacott and Shumway-Cook, 2002; and Yogev-Seligmann et al., 2008). Thus, cognitive tasks such as verbal fluency (Dubost et al., 2006), fine-motor movements (Yang et al., 2007), and arithmetics (van Iersel et al., 2007) have been shown to alter gait characteristics ranging from walking velocity, over 
stride-variability in length, time, or width, to stride-asymmetry ((Dubost et al., 2006; Yang et al., 2007); van Iersel et al., 2007). While there is a growing literature on the effects of cognitive loading on gait characteristics as well as postural control little is known about its effects on the sense of agency for movements (neither for the arm nor the entire body). Importantly, the combination of gait agency paradigms with cognitive loading may be able to illustrate the effect of resource availability on predictive processing and the sense of agency with respect to potential changes in the internal model of sensorimotor control.

In conclusion, we discussed that predictive processing, as intrinsically used for sensorimotor control, plays an important role in the sense of agency including locomotion. Only by comparing the predicted state of our movements to their consequences can we attribute actions to the correct agent, allowing us in turn to separate ourselves from the environment and from other agents around us. The central monitoring theorem presents an appropriate framework to investigate the sense of agency from a sensorimotor perspective and experimental paradigms have successfully illustrated the limits of conscious access to sensorimotor control. However, the gait agency paradigms reviewed here suggest that extending this framework to account for continuous full-body movements such as locomotion is not trivial. In these studies, sensorimotor control was not used to directly compensate for spatio-temporal deviations and could change independently of the perceived sense of agency. Extending agency paradigms to include human locomotion will allow refining the central monitoring framework for the sense of agency and open up new avenues of research by linking approaches from the field of agency to bodily self-consciousness, path integration, and spatial navigation.

\section{Acknowledgments}

The authors are supported by the Swiss National Science Foundation (Sinergia Grant CRSII1-125135: Balancing Self and Body).

\section{References}

Agid, Y., Blin, J., 1987. Nerve cell death in degenerative diseases of the central nervous system: clinical aspects. Ciba Foundation Symposium 126, 3-29.

Alexander, G.E., DeLong, M.R., Strick, P.L., 1986. Parallel organization of functionally segregated circuits linking basal ganglia and cortex. Annual Review of Neuroscience 9, 357-381.

Armstrong, D.M., 1988. The supraspinal control of mammalian locomotion. Journal de Physiologie 405, 1-37.

Asai, T., Tanno, Y., 2008. Highly schizotypal students have a weaker sense of self-agency. Psychiatry and Clinical Neurosciences 62, 115-119.

Blakemore, S.J., Goodbody, S.J., Wolpert, D.M., 1998a. Predicting the consequences of our own actions: the role of sensorimotor context estimation. Journal of Neuroscience $18,7511-7518$.

Blakemore, S.J., Wolpert, D.M., Frith, C.D., 1998b. Central cancellation of self-produced tickle sensation. Nature Neuroscience 1, 635-640.

Blakemore, S.J., Wolpert, D., Frith, C., 2000. Why can't you tickle yourself? Neuroreport 11, R11-R16.

Blakemore, S.J., Frith, C.D., Wolpert, D.M., 2001. The cerebellum is involved in predicting the sensory consequences of action. Neuroreport 12, 1879-1884.

Blakemore, S.J., Wolpert, D.M., Frith, C.D., 2002. Abnormalities in the awareness of action. Trends in Cognitive Sciences 6, 237-242.

Blanc, Y., Balmer, C., Landis, T., Vingerhoets, F., 1999. Temporal parameters and patterns of the foot roll over during walking: normative data for healthy adults. Gait \& Posture 10, 97-108.

Blanke, O., Metzinger, T., 2009. Full-body illusions and minimal phenomenal selfhood. Trends in Cognitive Sciences 13, 7-13.

Bridgemen, B., Kirch, M., Sperling, A., 1981. Segregation of cognitive and motor aspects of visual function using induced motion. Perception \& Psychophysics 29, 336-342.

Browner, N., Giladi, N., 2010. What can we learn from freezing of gait in Parkinson's disease? Current Neurology and Neuroscience Reports 10, 345-351.

Castiello, U., Paulignan, Y., Jeannerod, M., 1991. Temporal dissociation of motor responses and subjective awareness. A study in normal subjects. Brain 114 (Pt 6), 2639-2655.

Cunnington, R., Windischberger, C., Robinson, S., Moser, E., 2006. The selection of intended actions and the observation of others' actions: a time-resolved fMRI study. Neurolmage 29, 1294-1302.
Cutting, J.E., Moore, C., Morrison, R., 1988. Masking the motions of human gait. Perception \& Psychophysics 44, 339-347.

Daprati, E., Sirigu, A., 2002. Laterality effects on motor awareness. Neuropsychologia 40, 1379-1386

Daprati, E., Franck, N., Georgieff, N., Proust, J., Pacherie, E., Dalery, J., Jeannerod, M. 1997. Looking for the agent: an investigation into consciousness of action and self-consciousness in schizophrenic patients. Cognition 65, 71-86.

David, N., Bewernick, B.H., Cohen, M.X., Newen, A., Lux, S., Fink, G.R., Shah, N.J., Vogeley, K., 2006. Neural representations of self versus other: visual-spatial perspective taking and agency in a virtual ball-tossing game. Journal of Cognitive Neuroscience $18,898-910$.

David, N., Newen, A., Vogeley, K., 2008. The "sense of agency" and its underlying cognitive and neural mechanisms. Consciousness and Cognition 17, 523-534.

Drew, T., Prentice, S., Schepens, B., 2004. Cortical and brainstem control of locomotion. Progress in Brain Research 143, 251-261.

Dubost, V., Kressig, R.W., Gonthier, R., Herrmann, F.R., Aminian, K., Najafi, B., Beauchet O., 2006. Relationships between dual-task related changes in stride velocity and stride time variability in healthy older adults. Human Movement Science 25, 372-382.

Eccles, J.C., 1989. Evolution of the hominid brain: Bipedality; agility. In: Eccles, J.C. (Ed.), Evolution of the Brain: Creation of the self. Routledge, London, UK, pp. 36-69.

Ehrsson, H.H., 2007. The experimental induction of out-of-body experiences. Science 317, 1048.

Enzensberger, W., Fischer, P.A., 1996. Metronome in Parkinson's disease. Lancet 347, 1337.

Faisal, A.A., Selen, L.P., Wolpert, D.M., 2008. Noise in the nervous system. Nature Reviews Neuroscience 9, 292-303.

Farrer, C., Frith, C.D., 2002. Experiencing oneself vs another person as being the cause of an action: the neural correlates of the experience of agency. Neurolmage 15 596-603.

Farrer, C., Franck, N., Georgieff, N., Frith, C.D., Decety, J., Jeannerod, M., 2003. Modulating the experience of agency: a positron emission tomography study. Neurolmage 18, 324-333.

Feinberg, T.E., Schindler, R.J., Flanagan, N.G., Haber, L.D., 1992. Two alien hand syndromes. Neurology 42, 19-24.

Fink, G.R., Marshall, J.C., Halligan, P.W., Frith, C.D., Driver, J., Frackowiak, R.S., Dolan, R.J., 1999. The neural consequences of conflict between intention and the senses. Brain 122 (Pt 3), 497-512.

Fourneret, P., Jeannerod, M., 1998. Limited conscious monitoring of motor performance in normal subjects. Neuropsychologia 36, 1133-1140.

Fourneret, P., Franck, N., Slachevsky, A., Jeannerod, M., 2001. Self-monitoring in schizophrenia revisited. Neuroreport 12, 1203-1208.

Fourneret, P., Paillard, J., Lamarre, Y., Cole, J., Jeannerod, M., 2002. Lack of conscious recognition of one's own actions in a haptically deafferented patient. Neuroreport 13, 541-547.

Franck, N., Farrer, C., Georgieff, N., Marie-Cardine, M., Dalery, J., d'Amato, T., Jeannerod, M. 2001. Defective recognition of one's own actions in patients with schizophrenia. The American Journal of Psychiatry 158, 454-459.

Frith, C., 2005. The self in action: lessons from delusions of control. Consciousness and Cognition 14, 752-770.

Frith, C.D., Blakemore, S., Wolpert, D.M., 2000a. Explaining the symptoms of schizophrenia: abnormalities in the awareness of action. Brain Research 31, 357-363.

Frith, C.D., Blakemore, S.J., Wolpert, D.M., 2000b. Abnormalities in the awareness and control of action. Philosophical Transactions of the Royal Society of London. Series B, Biological Sciences 355, 1771-1788.

Fukuyama, H., Ouchi, Y., Matsuzaki, S., Nagahama, Y., Yamauchi, H., Ogawa, M., Kimura, J., Shibasaki, H., 1997. Brain functional activity during gait in normal subjects: a SPECT study. Neuroscience Letters 228, 183-186.

Glasauer, S., Stein, A., Gunther, A.L., Flanagin, V.L., Jahn, K., Brandt, T., 2009. The effect of dual tasks in locomotor path integration. Annals of the New York Academy of Sciences 1164, 201-205.

Grillner, S., Wallen, P., 1985. Central pattern generators for locomotion, with special reference to vertebrates. Annual Review of Neuroscience 8, 233-261.

Gwin, J.T., Gramann, K., Makeig, S., Ferris, D.P., 2011. Electrocortical activity is coupled to gait cycle phase during treadmill walking. NeuroImage 54, 1289-1296.

Hausdorff, J.M., 2005. Gait variability: methods, modeling and meaning. Journal of Neuroengineering and Rehabilitation 2, 19.

Helmholtz, H., 1866. Handbuch der Physiologischen Optik. Voss, Leipzig.

Hirsh, I.J., 1959. Auditory perception of temporal order. Journal of the Acoustical Society of America 31, 9.

Hirsh, I.J., Sherrick Jr., C.E., 1961. Perceived order in different sense modalities. Journal of Experimental Psychology 62, 423-432.

Jeannerod, M., 2003. The mechanism of self-recognition in humans. Behavioural Brain Research 142, 1-15.

Jeannerod, M., 2004. Visual and action cues contribute to the self-other distinction. Nature Neuroscience 7, 422-423.

Jeannerod, M., 2009. The sense of agency and its disturbances in schizophrenia: a reappraisal. Exp. Brain Res. 192, 527-532 (Electronic publication ahead of print 2008 Aug 16).

Jeannerod, M., Pacherie, E., 2004. Agency, simulation and self-identification. Mind \& Language 19, 34.

Kannape, O.A., Blanke, O., 2010. Conscious gait monitoring in virtual environments: Spatial, temporal and cognitive characteristics, Neuroscience 2010, 183.10/VV5 San Diego, USA.

Kannape, O.A., Schwabe, L., Tadi, T., Blanke, O., 2010. The limits of agency in walking humans. Neuropsychologia 48, 1628-1636.

Knoblich, G., Kircher, T.T., 2004. Deceiving oneself about being in control: conscious detection of changes in visuomotor coupling. Journal of Experimental Psychology. Human Perception and Performance 30, 657-666. 
Knoblich, G., Repp, B.H., 2009. Inferring agency from sound. Cognition 111, 248-262.

Knoblich, G., Stottmeister, F., Kircher, T., 2004. Self-monitoring in patients with schizophrenia. Psychological Medicine 34, 1561-1569.

Lenggenhager, B., Tadi, T., Metzinger, T., Blanke, O., 2007. Video ergo sum: manipulating bodily self-consciousness. Science 317, 1096-1099.

Leube, D.T., Knoblich, G., Erb, M., Grodd, W., Bartels, M., Kircher, T.T., 2003. The neural correlates of perceiving one's own movements. Neurolmage 20, 2084-2090.

Loomis, J.M., Klatzky, R.L., Golledge, R.G., Cicinelli, J.G., Pellegrino, J.W., Fry, P.A., 1993. Nonvisual navigation by blind and sighted: assessment of path integration ability. Journal of Experimental Psychology. General 122, 73-91.

Macellari, V., Giacomozzi, C., Saggini, R., 1999. Spatial-temporal parameters of gait: reference data and a statistical method for normality assessment. Gait \& Posture 10, 171-181.

Menzer, F., Brooks, A., Halje, P., Faller, C., Vetterli, M., Blanke, O., 2010. Feeling in control of your footsteps: Conscious gait monitoring and the auditory consequences of footsteps. Cognitive Neuroscience 1, 184-192.

Miall, R.C., Wolpert, D.M., 1996. Forward models for physiological motor control. Neural Networks 9, 1265-1279.

Miall, R.C., Weir, D.J., Wolpert, D.M., Stein, J.F., 1993. Is the cerebellum a smith predictor? Journal of Motor Behavior 25, 203-216.

Miyai, I., Tanabe, H.C., Sase, I., Eda, H., Oda, I., Konishi, I., Tsunazawa, Y., Suzuki, T., Yanagida, T., Kubota, K., 2001. Cortical mapping of gait in humans: a near-infrared spectroscopic topography study. NeuroImage 14, 1186-1192.

Moore, J.W., Ruge, D., Wenke, D., Rothwell, J., Haggard, P., 2010a. Disrupting the experience of control in the human brain: pre-supplementary motor area contributes to the sense of agency. Proceedings. Biological sciences 277, 2503-2509.

Moore, J.W., Schneider, S.A., Schwingenschuh, P., Moretto, G., Bhatia, K.P., Haggard, P., 2010b. Dopaminergic medication boosts action-effect binding in Parkinson's disease. Neuropsychologia 48, 1125-1132.

Morris, M.E., Iansek, R., Matyas, T.A., Summers, J.J., 1994. The pathogenesis of gait hypokinesia in Parkinson's disease. Brain 117 (Pt 5), 1169-1181.

Nielsen, T., 1963. Volition: a new experimental approach. Scandinavian Journal of Psychology 4, 6.

Nielsen, T., 1978. Acts. Analysis and Synthesis of Human Acting, Concerning the Subject and from the Standpoint of the Subject. Dansk Psykologisc Forlag, Copenhagen.

Nutt, J.G., Marsden, C.D., Thompson, P.D., 1993. Human walking and higher-level gai disorders, particularly in the elderly. Neurology 43, 268-279.

Posada, A., Franck, N., Augier, S., Georgieff, N., Jeannerod, M., 2007. Altered processing of sensorimotor feedback in schizophrenia. Comptes Rendus Biologies 330, 382-388.

Reed, C.L., Stone, V.E., Bozova, S., Tanaka, J., 2003. The body-inversion effect. Psychological Science 14, 302-308.

Repp, B.H., 2005. Sensorimotor synchronization: a review of the tapping literature. Psychonomic Bulletin and Review 12, 969-992.

Repp, B.H., 2006. Does an auditory distractor sequence affect self-paced tapping? Acta Psychologica 121, 81-107.

Repp, B.H., Knoblich, G., 2007. Toward a psychophysics of agency: detecting gain and loss of control over auditory action effects. Journal of Experimental Psychology. Human Perception and Performance 33, 469-482.

Roerdink, M., Bank, P.J., Peper, C.L., Beek, P.J., 2011. Walking to the beat of different drums: practical implications for the use of acoustic rhythms in gait rehabilitation. Gait \& Posture 33, 690-694.

Salomon, R., Szpiro-Grinberg, S., Lamy, D., 2011. Self-motion holds a special status in visual processing. PLoS One 6, e24347.

Sato, A., 2008. Action observation modulates auditory perception of the consequence of others' actions. Consciousness and Cognition 17, 1219-1227.

Sato, A., Yasuda, A., 2005. Illusion of sense of self-agency: discrepancy between the predicted and actual sensory consequences of actions modulates the sense of self-agency, but not the sense of self-ownership. Cognition 94, 241-255.
Schneider, K., 1955. Klinische Psychopathologie, 4., erweiterte Aufl. der Beiträge zur Psychiatrie. In: Thieme, G. (Ed.), . Stuttgart.

Shaw, F.E., 2002. Falls in cognitive impairment and dementia. Clinics in Geriatric Medicine 18, 159-173.

Shimada, S., Qi, Y., Hiraki, K., 2010. Detection of visual feedback delay in active and passive self-body movements. Experimental Brain Research 201, 359-364.

Slachevsky, A., Pillon, B., Fourneret, P., Pradat-Diehl, P., Jeannerod, M., Dubois, B., 2001. Preserved adjustment but impaired awareness in a sensory-motor conflict following prefrontal lesions. Journal of Cognitive Neuroscience 13, 332-340.

Slachevsky, A., Pillon, B., Fourneret, P., Renie, L., Levy, R., Jeannerod, M., Dubois, B., 2003. The prefrontal cortex and conscious monitoring of action: an experimental study. Neuropsychologia 41, 655-665.

Sperry, R.W., 1950. Neural basis of the spontaneous optokinetic response produced by visual inversion. Journal of Comparative and Physiological Psychology 43, $482-489$

Synofzik, M., Thier, P., Lindner, A., 2006. Internalizing agency of self-action: perception of one's own hand movements depends on an adaptable prediction about the sensory action outcome. Journal of Neurophysiology 96, 1592-1601.

Synofzik, M., Vosgerau, G., Newen, A., 2008. Beyond the comparator model: a multifactorial two-step account of agency. Consciousness and Cognition 17, 219-239.

Thaut, M.H., McIntosh, G.C., Rice, R.R., 1997. Rhythmic facilitation of gait training in hemiparetic stroke rehabilitation. Journal of the Neurological Sciences 151, 207-212.

Todorov, E., Jordan, M.I., 2002. Optimal feedback control as a theory of motor coordination. Nature Neuroscience 5, 1226-1235.

Tsakiris, M., Haggard, P., 2005. The rubber hand illusion revisited: visuotactile integration and self-attribution. Journal of Experimental Psychology. Human Perception and Performance 31, 80-91.

van den Bos, E., Jeannerod, M., 2002. Sense of body and sense of action both contribute to self-recognition. Cognition 85, 177-187.

van Iersel, M.B., Ribbers, H., Munneke, M., Borm, G.F., Rikkert, M.G., 2007. The effect of cognitive dual tasks on balance during walking in physically fit elderly people. Archives of Physical Medicine and Rehabilitation 88, 187-191.

Varraine, E., Bonnard, M., Pailhous, J., 2002. Interaction between different sensory cues in the control of human gait. Experimental Brain Research 142, 374-384.

Vogeley, K., Fink, G.R., 2003. Neural correlates of the first-person-perspective. Trends in Cognitive Sciences 7, 38-42.

von Holst, E., Mittelstaedt, H., 1950. Das Reafferenzprinzip. Die Naturwissenschaften $20,13$.

Watanabe, J., Ando, H., 2010. Pace-sync shoes: intuitive walking-pace guidance based on cyclic vibro-tactile stimulation for the foot. Virtual Reality 14, 7.

Weiskrantz, L., Elliott, J., Darlington, C., 1971. Preliminary observations on tickling oneself. Nature 230, 598-599.

Wolbers, T., Hegarty, M., 2010. What determines our navigational abilities? Trends in Cognitive Sciences 14, 138-146.

Wolpert, D.M., Ghahramani, Z., 2000. Computational principles of movement neuroscience. Nature Neuroscience 3, 1212-1217 (Suppl).

Wolpert, D.M., Ghahramani, Z., Jordan, M.I., 1995. An internal model for sensorimotor integration. Science 269, 1880-1882.

Wolpert, D.M., Diedrichsen, J., Flanagan, J.R., 2011. Principles of sensorimotor learning. Nature Reviews Neuroscience 12, 739-751.

Woollacott, M., Shumway-Cook, A., 2002. Attention and the control of posture and gait: a review of an emerging area of research. Gait \& Posture 16, 1-14.

Yang, Y.R., Chen, Y.C., Lee, C.S., Cheng, S.J., Wang, R.Y., 2007. Dual-task-related gait changes in individuals with stroke. Gait \& Posture 25, 185-190.

Yogev-Seligmann, G., Hausdorff, J.M., Giladi, N., 2008. The role of executive function and attention in gait. Movement Disorders 23, 329-342. 